\title{
Highlights of antimicrobial use in osteomyelitis as biofilms disease prototype in Venezuela
}

\section{Commentary}

Biofilms disease is a therapeutic challenge in daily medical practice, and requires the surgical support in cases such as bone infections, where the removal of devitalized tissue and infectious load if necessary, as well as achieve show bleeding with active perfusion in the bed that is creating are factors of paramount importance. Biofilms disease has numerous defense mechanism against the use of antimicrobials, since its inactivation, disinfection resistance, decreased perfusion, decreased bacterial metabolism, expression of heat shock proteins, among others. ${ }^{1}$

In our facility we specialize in handling complex diseases such as severe diabetic foot infections, which occur with large secondary vascular alterations mainly deficit arterial blood flow (peripheral arterial occlusive disease), and venous (chronic venous insufficiency), which coupled with repeated trauma from diabetic neuropathy, and immune deficit opsonization and phagocytosis by high levels of glycosylation, they are suitable bed for infection by capsulated microorganisms or bacteria producers exopolymers, which end up becoming the disease-causing biofilms, whose prototype is diabetic foot osteomyelitis. The use of monotherapies is contraindicated in these infections, that requires antimicrobial agents with high bactericidal capacity and ability to reduce adhesion and integrity of the biofilm, administered for longer than 4 weeks, ${ }^{2}$ plus able to penetrate and distributed in the same with a homogeneous pattern that reaches all the cells of the biofilm biomass. ${ }^{3}$

Drugs available in Venezuela and which have proved useful in osteomyelitis as biofilms disease are Daptomycin, Moxifloxacin, Levofloxacin, Clarithromycin, Azithromycin and Rifampin. In our experience the best antimicrobial combination in the management of diabetic foot related osteomyelitis, at the initial phase of attack for 3 weeks, Daptomycin at higher-doses (8 to $12 \mathrm{mg} / \mathrm{kg} / \mathrm{IV} / \mathrm{OD})^{4}$ + Rifampin $(600 \mathrm{mg} / \mathrm{PO} / \mathrm{OD})^{5}$ followed by 3 additional weeks of Linezolid (600mg/IV or PO/BID) + Rifampin (600mg/PO/OD) ${ }^{6}$ ideally starting 2 weeks prior to the osteotomy; and then continuing with 6 weeks of ambulatory antimicrobial consolidation therapy with a quinolone like Moxifloxacin (400mg/PO/OD) or Levofloxacin $(500 \mathrm{mg} / \mathrm{PO} / \mathrm{OD})+$ Rifampin to the already mentioned dose. ${ }^{7}$ But, if patient conditions allow the use of Daptomycin combined with Rifampin for the initial 6 weeks of antimicrobial therapy (attack phase), this is the more desirable and optimal scheme. It must make weekly monitoring CPK levels while using Daptomycin, and weekly blood counts with ESR, CPR, transaminases, GGT and alkaline phosphatase throughout treatment to monitor clinical outcomes and adverse events. X-ray films should be performed with a 3-weeks interval.

Microbiology of biofilms disease is critical. Bone sample taken at osteotomy was evaluated for conventional pyogenic bacteria and biofilm production capacity using conventional culture media, sonication and specific culture media and stains for the detection of biofilm formation. ${ }^{8}$ In cases where biofilm production demonstrated by

\author{
Marcel Marcano Lozada, ${ }^{1,2}$ Silvia Molero \\ Leon $^{3}$ \\ 'Medical Microbiologist, Medical Microbiology Unit Head, Angios \\ Vascular Center \& Wound Clinic, Caracas, Venezuela \\ ${ }^{2}$ Aggregate Professor of Microbiology, Medicine Faculty, \\ Universidad Central de Venezuela, Caracas, Venezuela \\ ${ }^{3}$ Internal Medicine Specialist, Angios Vascular Center \& Wound \\ Clinic, Caracas, Venezuela
}

Correspondence: Marcel Marcano-Lozada, MD. Medical Microbiology Unit, Angios Vascular Center \& Wound Clinic, Caracas,Venezuela, Email marcelmarcano@gmail.com

Received: July 01, 2016 | Published: July 05, 2016

Gram-positive cocci, is recommended to keep Daptomycin during the first 6 weeks (attack phase), always combined with Rifampin. In cases where it was shown to Gram-negative intestinal rod such as Klebsiella $s p$ : E. coli or Serratia marcescens or a Non-fermentative bacilli such as Pseudomonas aeruginosa, the switch to a Quinolone (if sensibility was demonstrated) or Carbapenem (Ertapenem -not for P. aeruginosaor Meropenem) over the Lipopeptide or the Ozaxolidinone must be immediately realized, moreover, in case of biofilm-positive strains, with the current use of Rifampin. The use of Macrolides should be limited to cases when Rifampin-resistant strains appears.

We prefer the use of Daptomycin over Linezolid in the diabetic foot osteomyelitis, because bactericidal activity of Daptomycin is greater and faster than Linezolid, with a minimum opportunity to develop antimicrobial resistance, moreover, bactericidal Daptomycin MoA not increases inflammatory mediators, because bacterial cell wall remains intact, the cellular death goes by an ionic channel formation on citoplasmatic membrane that leads to an imbalance in electrolites that finally disrupt metabolic pathways, nuclear acid \& protein synthesis and bacterial wall formation. ${ }^{9}$ In our experience (data on file), overall treatment success rate in Daptomycin-contain regimens for chronic osteomyelitis specially in the diabetic foot was greater than $75 \%$, with a very high safety profile of mild adverse events (fatigue), which not forced discontinuation of the drug in any patient (further analysis showed no causality in any case).

\section{Acknowledgments}

None.

\section{Conflicts of interest}

Authors declare there are no conflicts of interest.

\section{References}

1. Huang CT, Yu FP, McFeters GA, et al. Non-uniform spatial patterns of respiratory activity within biofilms during disinfection. Appl Environ Microbiol. 1995;61(6):2252-2256. 
2. Lipsky BA, Berendt AR, Cornia PB, et al. Infectious Diseases Society of America Clinical Practice Guideline for the Diagnosis and Treatment of Diabetic Foot Infections. Clin Infect Dis. 2012;54(12):e132-e173.

3. Cremieux AC, Mghir AS, Bleton R, et al. Efficacy of sparfloxacin and autoradiographic diffusion pattern of [14C]Sparfloxacin in experimental Staphylococcus aureus joint prosthesis infection. Antimicrob Agents Chemother. 1996;40(9):2111-2116.

4. Lamp KC, Friedrich LV, Mendez-Vigo L, et al. Clinical experience with daptomycin for the treatment of patients with osteomyelitis. Am J Med. 2007;120(10 Suppl 1):S13-S20.

5. John AK, Baldoni D, Haschke F, et al. Efficacy of daptomycin in implant-associated infection due to methicillin-resistant Staphylococcus aureus: Importance of combination with rifampin. Antimicrob Agents Chemother. 2009;53(7):2719-2724.
6. Baldoni D, Haschke M, Rajacic Z, et al. Linezolid alone or combined with rifampin against methicillin-resistant Staphylococcus aureus in experimental foreign-body infection. Antimicrob Agents Chemother. 2009;53(3):1142-1148.

7. Seaton RA, Gonzalez-Ramallo VA, Prisco V, et al. Daptomycin for outpatient parenteral antibiotic therapy: a European registry experience. Int J Antimicrob Agents. 2013;41(5):468-472.

8. Trampuz A, Piper KE, Jacobson MJ, et al. Sonication of removed hip and knee prostheses for diagnosis of infection. $N$ Engl J Med. 2007;357(7):654-663.

9. Novartis Europharm Ltd. Cubicin (daptomycin). Summary of Product Characteristics; 2009. 